9. LANGRIDGE (W.P.), KERNAGAN (R.J.), GLOVER (P.E.). A review of recent knowledge of the ecology of the main vectors of trypanosomiasis. Bull. Wld Hlth Org., 1963, 28 : 671-901.

10. LOSOS (G.J.). Trypanosomiasis. In : Infectious tropical disease of domestic animals. London, Longman scientific and technical, 1986. p. 183 318

11. Macl.FNNAN (K.J.R.). The epizootiology of trypanosomiasis in livestock in West Africa. In : MULLIGAN (H.W.), POTTS (W.H.), Eds. The African trypanosomiases. London, G. Allen and Unwin, 1970. p. 751-765.

12. MURRAY (M.), GRAY (A.R.). The current situation on animal trypanosomiasis in Africa. Prev. vet. Med., 1984, 2 : 30-32.

13. WOO (P.T.K.). Evaluation on the haematocrit centrifuge and other techniques for the field diagnosis of trypanosomiasis. Acta trop., 1969, 28 298-303.

DANIEL (A.D.), DADAH (A.J.), KALEJAIYE (J.O.), DALHATU (A.D.). Prevalence of bovine trypanosomosis in Gongola State of Northern Nigeria. Revue Élev. Méd. vét. Pays trop., 1993, 46 (4) : 571-574

A study of bovine trypanosomosis was carried out in Gongola State (Northern Nigeria), a supposedly tsctsc-frce region during the months of April-July, 1990. Blood samples were collected from 1,065 cattle and examined by buffy coat and stained smear methods. Packed red cell volume (PCV) was determined simultaneously. Fourty-two (3.9\%) of the animals examined were infected with trypanosomes. T. vivax was more frequently encountered than other species. Infection rate was high in the tsetse flies as 27 (22.7\%) of Glossina tachinoides caught, dissceted and examined were positive for $T$. vivax and $T$. congolense infections. Further studies are desirable in areas claimed tsetse-free in Nigeria.

Key words : Cattle - Trypanosomosis -Trypanosoma congolense. Trypanosoma vivax - Glossina tachinoides - Laboratory diagnosis Serology - Prevalence - Nigeria.

\section{La trypanosomose à Trypanosoma evansi (Steel 1885), Balbiani 1888 chez les petits ruminants de Mauritanie : Résultats d'inoculation expérimentale
et d'enquêtes sur le terrain}

\author{
P. Jacquiet ${ }^{1}$ \\ D. Cheikh ${ }^{1}$
}

A. Thiam ${ }^{1}$

\section{M.L. Dia ${ }^{1}$}

JACQUIET (P.), CHEIKH (D.), THIAM (A.), DIA (M.L.). La trypanosomose à Trypanosoma evansi (Steel 1885), Balbiani 1888 chez les petits ruminants de Mauritanie : Résultats d'inoculation expérimentale et d'enquêtes sur le terrain. Revue Élev. Méd. vét. Pays trop., 1993, 46 (4) : 574-578

Afin de préciser le rôle éventuel des petits ruminants dans l'épidémiologie de la trypanosomose à Trypanosoma evansi dans le Sud mauritanien, on a procédé à l'inoculation expérimentale d'une brebis et d'une chèvre par une souche locale de T. evansi isolée d'une chamelle laitière, et à des enquêtes dans des troupeaux de petits ruminants du Sud Trarza qui cotoient au pâturage des troupeaux de dromadaires souvent très contaminés. L'inoculation expérimentale a permis de confirmer que la brebis et la chèvre (races locales) sont réceptives. Seule la brebis a manifesté une sensibilité (amaigrissement et avortement). Pendant 220 jours après inoculation, le sang de la chèvre est resté infectant pour la souris. Chez la brebis, en revanche, on a pu constater l'alternance de phases infectantes et de phases "silencieuses". Sur le terrain, 207 frottis sanguins de caprins et 174 frottis d'ovins sont négatifs ainsi que 109 sérums de caprins et 78 d'ovins. Il semble donc que les petits ruminants ne jouent aucun rôle dans l'épidéniologie de la tiypanosonuse à $T$, evansi et ce, en dépit de leur réceptivité à l'inoculation expérimentale.

Mots clés : Ovin - Caprin - Trypanosomose - Trypanosoma evansi Enquête pathologique - Inoculation - Sérologie - Immunofluorescence indirecte - Épidémiologie - Mauritanie.

\section{Introduction}

La trypanosomose cameline à Trypanosoma evahsi est une contrainte majeure de l'élevage camelin dans le Sud Mauritanien. Dans le Sud Trarza (région du fleuve Sénégal) les taux d'infection globaux atteignent 15 p. 100 par frottis sanguins et 40 p. 100 en sérologie par immunofluorescence indirecte (1). Or, dans cette zone il est fréquent de voir des troupeaux de petits ruminants mixtes (ovins et caprins) pâturer avec les dromadaires.

Divers auteurs $(2,3,4,5,8)$ signalent la réceptivité des ovins et des caprins à $T$, evansi après inoculation expérimentale mais le rôle des petits ruminants dans l'épidémiologie de la trypanosomose à $T$. evansi a été très peu étudié sur le terrain. Ce travail a eu pour buts :

1. Centre national d'élevage et de recherches vétérinaires (CNERV), service de Parasitologie, BP 167 Nouakchott, République Islamique de Mauritanie.

Reçu le 13.7.1993, accepté le 19.10.1993. 
- d'inoculer expérimentalement une souche locale de $T$. evansi, isolée d'une chamelle, à une brebis et à une chèvre, puis d'effectuer un suivi parasitologique, sérologique et clinique de ces animaux ;

- d'enquêter sur le terrain, dans le Sud Trarza, sur les troupeaux de petits ruminants sédentaires qui côtoient toute l'année des troupeaux de dromadaires fortement infectés.

\section{Matériels et Méthodes}

\section{Inoculation expérimentale au laboratoire}

\section{Souche de Trypanosoma evansi}

Une souche de Trypanosoma evansi a été isolée en juillet 1990 d'une chamelle laitière en accès clinique aigu dans la vallée du fleuve Sénégal. Cette souche a été isolée puis entretenue sur lapins.

\section{Animaux en infestation expérimentale}

Une brebis et une chèvre originaires du Sud Trarza ont été régulièrement contrôlées avant inoculation pendant deux mois par frottis sanguins, inoculation à la souris et sérologie (immunofluorescence indirecte). Tous ces examens préalables se sont révélés négatifs. Ces animaux reçoivent une nourriture abondante, équilibrée et font l'objet de traitements anthelminthiques réguliers (fenbendazole, $10 \mathrm{mg} / \mathrm{kg}$ ).

\section{Inoculation}

La parasitémie à $T$. evansi des lapins étant toujours très faible, un passage préalable à la souris a été pratiqué. Vingt cinq millions de trypanosomes provenant du sang de la souris et dilués dans du sérum physiologique sont inoculés par injection intra-veineuse à la jugulaire de chacun des deux petits ruminants.

\section{Suivi des animaux}

Après prélèvement de sang sur anticoagulant (EDTA), la parasitémie est contrôlée de trois manières différentes:

- un frottis sanguin, fixé au méthanol et coloré au Giemsa - une concentration par centrifugation en tube hématocrite (9) ;

- une inoculation intrapéritonéale à la souris blanche.

Pour la sérologie, le sang est régulièrement prélevé sur tube siliconé. La méthode sérologique employée est l'immunofluorescence indirecte. Les lames d'antigène sont préparées sur place à partir du sang de souris en très forte parasitémie. La même souche de $T$. evansi est utilisée pour la confection des lames d' antigène et pour l'inoculation expérimentale. Les sérums de la brebis et de la chèvre sont dilués du $1 / 10$ e au $1 / 1280$ e. Les conjugués utilisés sont des conjugués anti-lgG de mouton et de chèvre (Sigma). L'observation en fluorescence est réalisée sur un microscope Olympus BH2-RFCA.

L'étude clinique concerne essentiellement la recherche des symptômes suivants : inappétence, démarches ébrieuses, prostration et avortement.

\section{Enquêtes sur le terrain}

Des troupeaux mixtes (ovins et caprins), sédentaires, pâturant dans les mêmes zones que les dromadaires souvent très infectés, sont choisis pour l'étude sur le terrain. Seuls les animaux âgés, ayant déjà connu un hivernage (saison où les populations de Tabanides et d'Hippoboscidés sont les plus denses) sont prélevés. Trois cent quatre vingt-un frottis sanguins (207 caprins, 174 ovins) sont réalisés puis colorés au Giemsa. Parmi ces animaux, 109 caprins et 78 ovins font l'objet d'analyses sérologiques ; les sérums de la brebis et de la chèvre inoculées expérimentalement servant de sérums témoins positifs.

\section{Résultats}

\section{Inoculation expérimentale}

\section{La brebis (tabl. I, fig. 1)}

Parmi tous les frottis sanguins réalisés, seul celui réalisé à $J_{6}$ est positif ( 1 trypanosome par champ microscopique au grossissement 400 ), tous les suivants sont négatifs: La technique de concentration en tube hématocrite est un peu plus sensible : elle reste positive - mais par intermittence - jusqu' à $\mathrm{J}_{41}$. Au delà, elle demeure constamment négative.

Les résultats de l'inoculation à la souris font apparaître une alternance de phases pendant lesquelles le sang est infectant pour la souris, et de phases "silencieuses" où l'inoculation à la souris se solde par un échec. Par cette méthode, on constate que la brebis conserve $T$. evansi pendant au moins 220 jours.

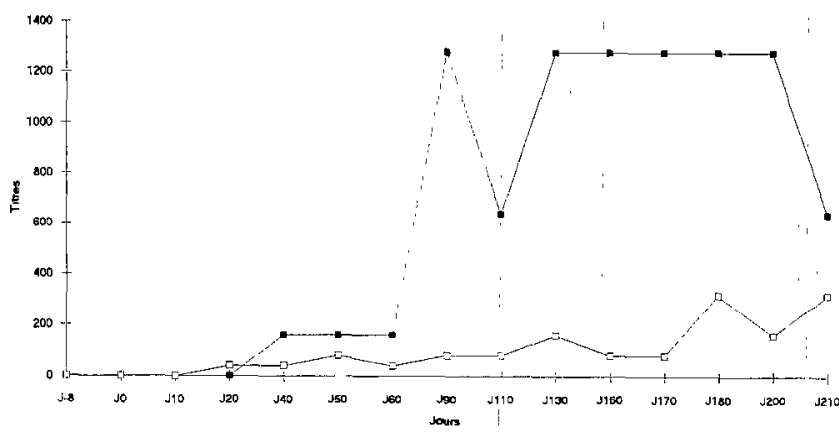

Figure 1 : Dosage des $\operatorname{Ig} G$ en immunofluorescence indirecte chez la brebis ( $\square$ ) et la chèvre ( $\square$ ) infectées experimentalement par T. evansi. 


\section{Communication}

TABLEAU I Suivi de la brebis après inoculation intraveineuse de 25 millions de trypanosomes(Trypanosoma evansi)

\begin{tabular}{|c|c|c|c|c|c|}
\hline Contrôle & Frottis & Concentration & Inoculation & $\begin{array}{l}\text { Faiblesse, } \\
\text { inappétence }\end{array}$ & $\begin{array}{l}\text { Tournis et } \\
\text { démarche } \\
\text { ébrieuse }\end{array}$ \\
\hline 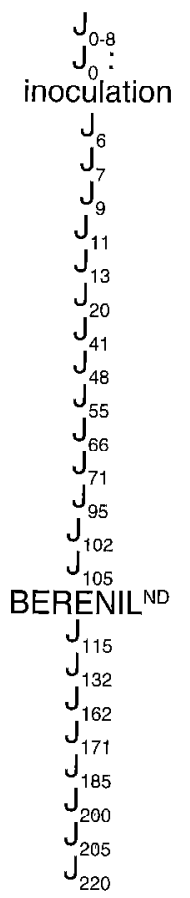 & $\begin{array}{l}0 \\
0 \\
\\
+ \\
0 \\
0 \\
0 \\
0 \\
0 \\
0 \\
0 \\
0 \\
0 \\
0 \\
0 \\
0 \\
0 \\
\\
0 \\
0 \\
0 \\
0 \\
0 \\
0 \\
0 \\
0\end{array}$ & $\begin{array}{l}0 \\
0 \\
\end{array}$ & $\begin{array}{c}0 \\
0 \\
\\
+ \\
\text { N.E. } \\
\text { N.E. } \\
\text { N.E. } \\
\text { N.E. } \\
+ \\
\text { N.E. } \\
\text { N.E. } \\
\text { N.E. } \\
0 \\
0 \\
+ \\
\text { N.E. } \\
0 \\
\\
0 \\
0 \\
0 \\
\text { N.E. } \\
0 \\
\text { N.E. } \\
0 \\
+\end{array}$ & $\begin{array}{l}0 \\
0 \\
+ \\
+ \\
+ \\
+ \\
0 \\
0 \\
0 \\
0 \\
0 \\
0 \\
0 \\
0 \\
+ \\
+ \\
+ \\
\\
0 \\
0 \\
0 \\
0 \\
0 \\
0 \\
0 \\
0\end{array}$ & $\begin{array}{l}0 \\
0 \\
\\
+ \\
+ \\
+ \\
+ \\
0\end{array}$ \\
\hline
\end{tabular}

Concentration: : méthode de concentration en tube microhématocrite.

Inoculation : inoculation intrapéritonéale à la souris avec contrôles de la souris à $J_{3^{\prime}} J_{6^{\prime}} J_{9^{\prime}} J_{12^{\prime}} J_{15}$ et $J_{2\}^{\prime}}$
N.E. : non effectué.

Sur le plan sérologique, les IgG atteignent un niveau décelable entre $J_{30}$ et $J_{40}$. Au delà de $J_{90}$, les IgG persistent à des concentrations importantes (test positif à 1/1 280 e ) et ceci jusqu' à $J_{210}$. Cliniquement, on a pu constater deux périodes à symptomatologie nette :

- la première entre $J_{6}$ et $J_{10}$ avec inappétence, démarche ébrieuse et tournis. Tous ces symptômes ont régressé spontanément ;

- la deuxième, vers $\mathrm{J}_{65}$, avec amaigrissement prononcé, poil piqué, démarche ébrieuse et avortement. L'ampleur des symptômes a motivé une intervention thérapeuthique (BERENIIND, 3,5 mg/ $\mathrm{kg}$ ) qui a été suivie d'une amélioration nette très rapide $(24 \mathrm{~h})$.

Après ce traitement, aucune symptomatologie n'a été relevée alors que l'inoculation à la souris est positive à $\mathrm{J}_{210}$ : le traitement au BERENIIND, n'a donc pas "blanchi" la brebis.

\section{La chèvre (tabl. II , fig. 1)}

Le frottis sanguin est positif uniquement à $J_{6}$ (un seul trypanosome sur l'ensemble de la lame). La concentration en tube hématocrite est positive par intermittence et ceci jusqu'à $\mathrm{J}_{205}$. En revanche, à chaque fois que l'inoculation à la souris a été pratiquée entre $\mathrm{J}_{6}$ et $\mathrm{J}_{220}$, elle s'est révélée positive. On ne retrouve donc pas les phases "silencieuses" observées chez la brebis.

L'apparition des IgG chez la chèvre s'est faite très lentement $\left(1 / 80\right.$ e seulement à $\left.J_{50}\right)$. Leur niveau maximum $\left(1 / 320 \theta\right.$ à $\left.J_{180}\right)$ reste bien en dessous de celui retrouvé chez la brebis. Un très léger affaiblissement (inappétence et adynamie) a été constaté entre $J_{7}$ et $J_{9}$ qui a très rapidement et spontanément rétrocédé. A partir de $J_{11}$, plus aucune manifestation clinique n'a été mise en évidence chez la chèvre.

\section{Résultats d'enquêtes sur le terrain}

Frottis sanguins

Aucun des 381 frottis sanguins n'a révélé de trypanosomes. 
TABLEAU II Suivi de la chèvre après inoculation intraveineuse de 25 millions de trypanosomes(Trypanosoma evansi)

\begin{tabular}{|c|c|c|c|c|c|}
\hline Contrôle & Frottis & Concentration & Inoculation & $\begin{array}{l}\text { Faiblesse, } \\
\text { inappétence }\end{array}$ & $\begin{array}{l}\text { Tournis et } \\
\text { démarche } \\
\text { ébrieuse }\end{array}$ \\
\hline $\begin{array}{c}J_{0-8} \\
J_{0}^{0-} \\
\text { inoculation } \\
J_{6} \\
J_{7}^{6} \\
J_{9} \\
J_{11} \\
J_{13}^{13} \\
J_{20} \\
J_{25} \\
J_{41}^{J_{15}} \\
J_{48} \\
J_{55}^{5} \\
J_{66} \\
J_{05} \\
J_{102}^{102} \\
J_{115} \\
J_{132} \\
J_{162}^{162} \\
J_{171} \\
J_{185} \\
J_{200}^{200} \\
J_{205}^{205} \\
J_{210} \\
J_{220}\end{array}$ & $\begin{array}{l}0 \\
0 \\
+ \\
+ \\
0 \\
0 \\
0 \\
0 \\
0 \\
0 \\
0 \\
0 \\
0 \\
0 \\
0 \\
0 \\
0 \\
0 \\
0 \\
0 \\
0 \\
0 \\
0 \\
0 \\
0\end{array}$ & $\begin{array}{l}0 \\
0 \\
+ \\
0 \\
0 \\
0 \\
0 \\
0 \\
+ \\
0 \\
0 \\
0 \\
0 \\
0 \\
0 \\
0 \\
0 \\
0 \\
0 \\
0 \\
0 \\
+ \\
0 \\
0\end{array}$ & $\begin{array}{c}0 \\
0 \\
+ \\
\text { N.E. } \\
\text { N.E. } \\
0 \\
\text { N.E. } \\
+ \\
\text { N.E. } \\
\text { N.E. } \\
+ \\
\text { N.E. } \\
+ \\
\text { N.E. } \\
+ \\
\text { N.E. } \\
+ \\
+ \\
\text { N.E. } \\
+ \\
+ \\
+ \\
\text { N.E. } \\
+\end{array}$ & $\begin{array}{l}0 \\
0 \\
0 \\
0 \\
+ \\
0 \\
0 \\
0 \\
0 \\
0 \\
0 \\
0 \\
0 \\
0 \\
0 \\
0 \\
0 \\
0 \\
0 \\
0 \\
0 \\
0 \\
0 \\
0\end{array}$ & $\begin{array}{l}0 \\
0 \\
0 \\
0 \\
0 \\
0 \\
0 \\
0 \\
0 \\
0 \\
0 \\
0 \\
0 \\
0 \\
0 \\
0 \\
0 \\
0 \\
0 \\
0 \\
0 \\
0 \\
0 \\
0\end{array}$ \\
\hline
\end{tabular}

Concentration : méthode de concentration en tube microhématocrite. Inoculation: inoculation intrapéritonéale à la souris avec contrôles de la souris à $J_{3^{\prime}} J_{6^{3}} J_{9}, J_{12}, J_{15}$ et $J_{2 t}$.
N.E. : non effectué.

\section{Sérologie en immunofluorescence indirecte}

Chez les caprins, 5 sérums (sur 109) ont donné une très faible fluorescence au $1 / 20$ e et 2 au 1/40e. Chez les ovins, un seul sérum sur 78 a révélé une très faible fluorescence au $1 / 80$ e. Tous les autres sérums sont négatifs.

\section{Discussion}

La brebis ef la chèvre se sont révélées réceptives à la souche locale de Trypanosoma evansi, isolée d' une chamelle.

Dans cette étude, la lecture de frottis sanguins et la technique de concentration en tube hématocrite sont très peu sensibles et de peu d'utilité dans la recherche de $T$. evansi chez les petits ruminants, compte tenu des parasitémies très faibles. L'inoculation à la souris est une méthode précieuse chez la chèvre ; en revanche, la présence de phases silencieuses chez la brebis (pendant lesquelles le parasite se trouverait en des localisations profondes : liquide céphalorachidien, ganglions ?), diminue la sensibilité de cette technique.

Les travaux de DENNIG (2) ont montré que les chèvres inoculées expérimentalement par $T$. evansi pouvaient développer soit une forme aiguë et mourrir, soit une forme chronique totalement asymptomatique. C'est ce dernier cas qu'on a rencontré avec une persistance du parasite d'au moins 220 jours.

La brebis a connu entre $J_{60}$ et $J_{110}$ un épisode clinique plus marqué, cependant l'évolution de l'infection après $\mathrm{J}_{120}$ et le traitement au BERENIL ${ }^{\mathrm{ND}}$ prend une allure chronique asymptomatique.

La lecture des frottis sanguins réalisés sur le terrain est de peu d'utilité, en revanche la sérologie par immunofluorescence indirecte (recherche des lgG) est un bon témoin de l'infection par $T$. evansi. Or, sur les animaux prélevés dans le Sud Trarza, il n'a été trouvé qu'un très petit nombre de cas douteux. II semble donc que les ovins et les caprins du Sud Trarza ne jouent qu'un rôle tout à fait marginal - sinon aucun - dans l'épidémiologie de la trypanosomose à $T$. evansi. De plus, les parasitémies enregistrées lors de l'inoculation expérimentale étant tellement faibles et transitoires, on peut douter de l'efficacité de la transmission mécanique dans le sens : petits ruminants vers dromadaire.

La réceptivité après inoculation expérimentale étant maintenant bien documentée $(2,3,4,5)$, comment peuton expliquer l'absence d'infection naturelle dans des 
zones où la transmission de la trypanosomose cameline est importante ? Cela pourrait provenir d'un manque d'attractivité des petits ruminants pour les Tabanides et/ou les Hippoboscidés vecteurs.

\section{Bibliograhie}

1. Centre National d'Elevage et de Recherches Vétérinaires (CNERV). Service de parasitologie. Rapport d'activités 1992. Nouakchott, R.I. Mauritanie, CNERV, 1993.

2. DENNIG (H.K.). La chèvre, réservoir potentiel de Trypanosoma evansi. Revue Méd. vél., 1989, 140 (8-9) : 763.

3. DIA (M.L.). Etude du pouvoir pathogène d' une souche de Trypanosoma evansi (Steel 1885 ), Balbiani 1888 isolée de Mauritanie et diagnostic sérologique de la trypanosomose expérimentale par immunofluorescence indirecte et immunoenzymologie (ELISA). Mémoire de maîtrise ès Sciences Vétérinaires. Ecole nationale vétérinaire de Lyon, 6 juillet 1992 , $62 \mathrm{p}$.

4. EI AMIN (A), ELAMIN (EA) Bioavailability of Rerenil ${ }^{\mathrm{ND}}$ (diminazène aceturate) in tissues of mice and goats and its effect on Trypanosoma evansi infections. In : Premier Séminaire International sur les Trypanosomoses animales non transmises par les glossines, Annecy (France), 14-16 octobre 1992.

5. ELAMIN (E.A.). Efficacy of diminazene aceturate (Berenil ${ }^{\mathrm{ND}}$ ) against experimental Trypanosoma evansi infections in goats. In : Premier Séminaire International sur les Trypanosomoses animales non transmises par les glossines, Annecy (France), 14-16 octobre 1992.

6. HOARE (C.A.). The trypanosomes of mammals : a zoological monograph. Oxford, Blackwell Scientific Publications, 1972. 749 p.

7. LUCKINS (A.G.). Evaluation of an indirect fluorescent antibody test, linked immunosorbent assay and quantification of immunoglobulins in the diagnosis of bovine trypanosomiasis. Trop. Anim. Hth Prod, 1978,10 : 149-159.

8. MAHMOUD (M.M.), GRAY (A.R.). Trypanosomiasis due to Trypanosoma evansi (Steel 1885), Balbiani 1888 : a review of recent research. Trop. Anim. Hlth Prod., 1980, 12 : 35-47.

9. WOO (P.T.K.). The haematocrit centrifuge technique for the diagnosis of African Trypanosomiasis. Acta trop., 1970, 27 : 384-386.

JACQUIET (P.), CHEIKH (D.), THIAM (A.), DIA (M.L.). Trypanosomosis due to Trypanosoma evansi (Steel 1885), Balbiani 1888 in small ruminants in Mauritania : experimental inoculation in the laboratory and field survey results. Revue Élev. Méd. vét. Pays trop., 1993, 46 (4) : 574578

In order to define the eventual role of small ruminants in the epidemiology of $T$. evansi infection in Southern Mauritania, the following exepriments were carried out : the intravenous inoculation of a ewe and a goat with a local strain of $T$ evansi isolated from a dairy camcl ; surveys of small ruminant flocks which graze with infected camels in the South of the Trarza region. The experimental inoculation allowed to show that local sheep and goats are receptive. Only the ewe showed a clinical episode with loss of weight and abortion. During 220 days after inoculation the blood of the goat remained constantly infectious for the mouse whereas in the same period the ewe's blood showed an alternation of infectious and non-infectious phases. However in the field, none of 381 blood smears of small ruminants (207 goats, 174 sheep) were positive and none of the 187 serums ( 109 goats, 78 sheep). Therefore, it seems that the small ruminants of the South Mauritania do not play any role in the epidemiology of $T$. evansi camel trypanosomosis even if they are receptive to experimental inoculation.

Key words : Goat - Sheep - Trypanosomosis - Trypanosoma evansi Pathological survey - Inoculation methods - Serology - Indirect immunofluorescence - Epidemiology - Máuritania.

\section{Presence of ketones in the serum of Trypanosoma evansi infected camels (Camelus dromedarius) in the Sudan}

\author{
Kamal S.M. Hussein ${ }^{1}$ \\ Gundi S. Gasmir ${ }^{1}$
}

HUSSEIN (Kamal S.M.), GASMIR (Gundi S.). Présence de cétones: dans le sérum de dromadaires (Camelus dromedarius) infectés par Trypanosoma evansi au Soudan. Revue Élev. Méd. vét. Pays trop., 1993, 46 (4) : 578-579

Cent échantillons sanguins et deux échantillons d'urine prélevés chez des dromadaires (Camelus dromedarius) ont été analysés au Laboratoire de Recherche vétérinaire de 1'Etat Oriental de Kassala, au Soudan, pour rechercher la présence de cétones. Les cinquante sérums provenant d'animaux infectés par des trypanosomes ont donné des résultats positifs. Sur les cinquante autres provenant de chameaux non infectés, 45 présentaient des résultats négatifs. Les cinq échantillons restant positifs, l'étaient également au test du chlorure de mercure.

Mots clés : Dromadaire - Camelus dromedarius - Trypanosomose animale - Trypanosoma evansi - Cétone - Sérum - Soudan.

\section{Introduction}

Camel owners in Eastern Sudan claim that in camel trypanosomes are associated with a characteristic pungent odour of the urine, just like the smell of bad water melon. HUNTER (2) detected a small amount of ketones in the urine of $T$. evansi positive camels. In the present study serum and urine samples were used for further elucidation of this feature.

\section{Materials and Methods}

Hundred blood samples were collected from the jugular vein at the Veterinary clinic or from different localities around Kassala town during the year 1990-1991. Blood was collected in vaccutainers containing EDTA and plain vaccutainers for serum separation.

Sera were kept at $-20^{\circ} \mathrm{C}$ until used. From each blood sample, wet film, thin smear and buffy coat (PCV) preparations were examined for detection of trypanosomes. All sera were examined by the mercuric chloride test. One gram of mercuric chloride was dissolved in $250 \mathrm{ml}$ of distilled water; $5 \mathrm{ml}$ from this solution was made up to 500 $\mathrm{ml}$ with distilled water. One $\mathrm{ml}$ of this solution was put into each of two test tubes, and $20 \mu \mathrm{l}$ of serum was added to one test tube and the other was left as a control. White turbidity indicated a positive reaction. According to these examinations, the sera were divided into three groups. In group I, the trypanosomes and mercuric chlori-

1. Eastern State Veterinary Research Laboratory, Kassala, POB 237, Soudan.

Reçu le 14.8.1992, accepté le 3.6.1993. 